\title{
On the possible variability of metallic-line and Mercury-Manganese stars ${ }^{\star}$
}

\author{
S.J. Adelman \\ Department of Physics, The Citadel, 171 Moultrie Street, Charleston, SC 29409, U.S.A.
}

Received September 6; accepted October 9, 1996

\begin{abstract}
Differential Strömgren uvby photometric observations from the $0.75-\mathrm{m}$ Four College Automated Photoelectric Telescope were obtained for three metallicline and three Mercury-Manganese stars. None were found to be variable. These sharp-lined stars may not be the best tests for class variability as their polar axes are pointed towards the earth. Each presents essentially the same visible hemisphere as it rotates.
\end{abstract}

Key words: stars: chemically peculiar

\section{Introduction}

The astronomical literature contains many papers about possible low amplitude photometric variability of the upper main sequence non-magnetic chemically peculiar stars: the metallic-line (Am) and the Mercury-Manganese (HgMn) stars. It is important either to confirm or to set limits on such claims as the results are crucial to our understanding these stars. This paper examines differential Strömgren uvby photometry obtained during the first six years (Sept. 1990 - July 1996) (year 1 is Sept. 1990July 1991, year 2 is Sept. 1991 - July 1992, etc.) of the 0.75-m Four College Automated Photoelectric Telescope (FCAPT) on Mt. Hopkins, AZ. The telescope measured the dark count and then in each filter the sky-ch-c-v-c$v-c-v-c$-ch-sky where sky is a reading of the sky, $c h$ that of the check star, $c$ that of the comparison star, and $v$ that of the variable star. By measuring two non-variable stars for each potentially variable star, one can derive an estimate of the accuracy of the photometry. This paper extends previous results for $53 \mathrm{Tau}, 68 \mathrm{Tau}, \mathrm{HR}$ 4072, and HR 6096 (Adelman 1993) as well as examines observations of $15 \mathrm{Vul}$ and o Peg obtained this past observing year.

Send offprint requests to: S.J. Adelman

* Tables 2-7 are available in electronic form at the CDS via anonymous ftp to cdsarc.u-strasbg.fr (130.79.128.5) or via http://cdsweb.u-strasbg.fr/Abstract.html
Table 1 gives information on each group of variable, comparison, and check stars (Hoffleit 1982). Tables 2-7 contain the new observations along with the yearly and total means and standard deviations. No corrections have been made for neutral density filter differences among each group of variable, comparison, and check stars.

Table 1. Group information

\begin{tabular}{llcll}
\hline \hline Star & Type & $V$ & $B-V$ & Spectral Type \\
\hline 68 Tau & $v$ & 4.29 & +0.05 & A2 IV \\
64 Tau & $c$ & 4.80 & +0.15 & A7 V \\
80 Tau & $c h$ & 5.58 & +0.32 & F0 V \\
& & & & \\
15 Vul & $v$ & 4.64 & +0.18 & A4 III \\
18 Vul & $c$ & 5.52 & +0.08 & A3 III \\
21 Vul & $c h$ & 5.18 & +0.18 & A7 IVn \\
& & & & \\
o Peg & $v$ & 4.79 & -0.01 & A1 IV \\
32 Peg & $c$ & 4.81 & -0.00 & B9 III \\
38 Peg & $c h$ & 5.63 & -0.10 & B9.5 V \\
& & & & \\
53 Tau & $v$ & 5.35 & -0.08 & B9 IV \\
HR 1375 & $c$ & 5.99 & -0.03 & B8 IV-V \\
51 Tau & $c h$ & 5.65 & +0.28 & F0 V \\
& & & & \\
HR 4072 & $v$ & 4.97 & -0.06 & A0p \\
32 UMa & $c$ & 5.75 & $\ldots$ & A8 III \\
HR 4215 & $c h$ & 6.39 & -0.02 & A1 V \\
HR 6096 & $v$ & 6.23 & +0.07 & B9 V \\
HR 6041 & $c$ & 6.25 & +0.13 & A1 V \\
$\sigma$ Ser & $c h$ & 4.82 & +0.34 & F0 V \\
\hline \hline
\end{tabular}

\section{The hot metallic-line (Am) stars}

Lanz \& Mathys (1990) discovered that the sharp-lined $\left(v \sin i=6 \mathrm{~km} \mathrm{~s}^{-1}\right.$ ) hot Am prototype star o Peg (spectral type A1 IV) has a magnetic field of order $2 \mathrm{kG}$ 
using the Stenflo \& Lindegren (1977) multiline and another technique. Recently Savanov \& Savalyeva (1996) used the former on the spectrum of the mild Am star $15 \mathrm{Vul}$ and derived a magnetic field of about $2.4 \mathrm{kG}$. Following the example of the magnetic CP stars, one might expect to observe spectral and light variability although in this case, the scale size of the field, which is not detected using a standard Zeeman analyzer (Babcock 1958), is expected to be much smaller and the field geometry much more complex. However, despite previous claims of low amplitude variability, Adelman (1993) showed that another prototype hot Am star 68 Tau was not variable within an observing season.

In years 1 and 2, 15 and 30 FCAPT uvby observations, respectively, were made of 68 Tau $(=$ HR $1389=$ HD 27962). A careful reexamination of that data shows that observation 12 of year 1 and observations 10 and 11 of year 2 should have been omitted. In year 3 I obtained 14 new observations (Table 2). There is no evidence of variability within a year. The data for year 3 has smaller errors than those for previous years and for each filter the $c h-c$ standard deviations of the means are equal or larger than those for $v$-c. The agreement of the $v$-c averages for years 2 and 3 is excellent. The largest difference between the $v$-c values for years 1 and 2 are for $v$ and $y$ and are about $1.5 \sigma$ which is within the expected errors. Thus 68 Tau is photometrically constant to within the errors of the data. It would be useful to get another year of data with the quality of year 3 and many more observations to reduce the upper limit on any variability. This is particularly important as 68 Tau is a blue straggler in the Hyades.

With the use of electronic detectors for spectroscopy and the resultant increase in the signal-to-noise ratios in the last decade or two, one can also use high dispersion spectra to set stricter limits on the spectral variability of supposedly non-variable stars. I found, for example, that the $2.4 \AA \mathrm{mm}^{-1}$ Dominion Astrophysical Observatory spectra of 68 Tau ( $67 \AA$ in length) obtained with a Reticon (pixel width $15 \mu$ ) used in the analysis of this star (Adelman 1994) agreed well in the regions of overlap to within the errors expected for spectra with signal-to-noise ratios of 200 and that $v \sin i=9 \mathrm{~km} \mathrm{~s}^{-1}$.

In the past year 43 uvby observations of $15 \mathrm{Vul}$ $(=$ HR $7653=$ HD 189849) were obtained (Table 3$)$. The standard deviation of the mean $v$-c for all four magnitudes is about 0.004 mag. while for the $v-c h$ and $c h-c$ values about 0.009 mag. Thus $15 \mathrm{Vul}$ is more stable than $21 \mathrm{Vul}$ and probably is constant. Bolcal et al. (1992) found it has rather mild abundance anomalies and $v \sin i=9 \mathrm{~km} \mathrm{~s}^{-1}$.

I obtained 23 and 2 differential uvby observations of o Peg (= HR $8641=$ HD 214994) (Table 4) in the fall of 1995 and the spring of 1996, respectively. The ch-c and $v-c$ star standard deviations about the mean for $v$, $b$, and $y$ are typically $0.003 \mathrm{mag}$. Both values for $u$ are
0.008 mag. Thus the variability of o Peg in the best observed filters $v, b$, and $y$ is at most of order 0.003 mag.

\section{The Mercury-Manganese (Hg-Mn) stars}

The possible variability of the Am stars lead me to reexamine and extend my results for the hotter "nonmagnetic" HgMn stars. Observations of 53 Tau, HR 4072, and HR 6096 (later shown to be a HgMn star by LopezGarcia \& Adelman 1994) made during the first two years of the FCAPT (Adelman 1993) and of $\alpha$ And (Adelman et al. 1994) made with the same telescope showed that these stars were constant. A reexamination of the data in the literature agreed with this conclusion.

After a reexamination of the data made during years 1 and 2 , there are respectively 6 and 17 good observations of 53 Tauri (= HR $1339=$ HD 27295) in Adelman (1993). During year 3, 18 observations were made (Table 5). There is no evidence for variability within a year. The averages for years 2 and 3 differ by about one-half the standard deviations of the means. Those values for year 1 usually are slightly more discrepant which suggests a slight difference in the atmospheric extinction.

Adelman (1993) reported on 24 uvby observations of HR 4072 (= HD 89822) made during year 2. Table 5 presents $6,36,51$, and 50 sets of values from years 3 , 4,5 , and 6, respectively. Except for the $v-c h$ and $c h-c$ values of $v$ for year 6 , the yearly means are quite close to one another and within each year there is no evidence of variability.

Adelman (1993) gives $24 u v$ and 12 by observations of HR 6096 (= HD 147550) made in year 1 and 26 uvby values for year 2 . Table 6 contains $6,16,26$, and $29 u v b y$ observations made during years $3,4,5$, and 6 , respectively. As for HR 4072, the $v-c, v$-ch, and $c h-c$ values for each year and magnitude are close to one another except for the $v$ ch and ch-c $v$ values for year 6 . For both stars these are offset by about 0.045 magnitudes suggesting that something happened to produce this offset, perhaps a change in the extinction or for both stars a change in the check star as the $v-c$ averages are consistent between years.

\section{Comments}

Although the Am stars have smaller true rotational velocities than the normal A stars (Abt \& Moyd 1973), o Peg, $68 \mathrm{Tau}$, and $15 \mathrm{Vul}$ being some of the most sharplined have one of their rotational poles pointed close to the line of sight. If photometric variability is due to different parts of the surface appearing in and disappearing from the observed hemisphere, this will only happen near the limb where limb darkening reduces the effect. If the changes in the surface composition and magnetic fields occur over a time long compared to the human life span as is thought based on various radiative diffusion scenarios, then these stars are among the worst cases to look for 
intrinsic variability among the Am stars. However, if this paradigm changes, then the lack of photometric variability for at least the sharp-lined Am stars acts as a constraint for alternative points-of-view.

Similar considerations apply to the HgMn stars. In both types of stars, searches for photometric variability should be concentrated on those stars with the largest values of $v \sin i$. It is also desirable to work with stars which have suitable photometric comparison stars.

Acknowledgements. This work was supported in part by NSF grant AST-9115114 to The Citadel and in part by grants from The Citadel Development Foundation. I appreciate the continuing efforts of Louis J. Boyd and Robert J. Dukes Jr., to keep the FCAPT operating properly.

\section{References}

Abt H.A., Moyd K.I., 1993, ApJ 182, 809

Adelman S.J., 1988, MNRAS 230, 671

Adelman S.J., 1993, A\&A 269, 411

Adelman S.J., 1994, MNRAS 271, 355

Adelman S.J., Brown B.H., Caliskan H., Reese D.F., Adelman C.J., 1994, A\&AS 106, 333

Babcock H.W, 1958, ApJS 3, 141

Bolcal C., Kocer D., Adelman S.J., 1992, MNRAS 258, 270

Hoffleit D., 1982, The Bright Star Catalogue, 4th edition, Yale University Observatory, New Haven, CT

Lopez-Garcia Z., Adelman S.J., 1994, A\&AS 107, 353

Mathys G., Lanz T., 1990, A\&A 230, L21

Savanov I.S., Savalyeva Y.Y., 1996, in M.A.S.S. Model Atmospheres and Spectrum Synthesis, 5th Vienna Workshop, Adelman S.J., Kupka F. and Weiss W.W. (eds.) ASP Conf. Ser. 108. San Francisco, ASP, p. 262

Stenflo J.O., Lindegren L., 1977, A\&A 59, 367 